\title{
Changing Mechanisms of Surface Relief and the Damage Evaluation of Low Cycle Fatigued Austenitic Stainless Steel
}

\author{
Nao Fujimura ${ }^{1, *}$, Takashi Nakamura ${ }^{1}$, and Kosuke Takahashi ${ }^{1}$ \\ ${ }^{1}$ Hokkaido University, Division of Mechanical and Space Engineering, Faculty of Engineering, Kita 13, Nishi 8, Kita-ku, Sapporo, \\ Hokkaido, Japan
}

\begin{abstract}
To quantitatively investigate the cause of the changes in arithmetic mean roughness $R_{\mathrm{a}}$ and arithmetic mean waviness $W_{\mathrm{a}}$ of austenitic stainless steel under low-cycle fatigue loading, precise observation focusing on persistent slip bands (PSBs) and crystal grain deformations was conducted on SUS316NG. During the fatigue tests, the specimen's surface topography was regularly measured using a laser microscope. The surface topographies were analysed by frequency analysis to separate the surface relief due to PSBs from that due to grain deformation. The height caused by PSBs and that by grain deformation were measured respectively. As a result, both of the heights rose with the increase of usage factor $(U F)$. The amount of increase in the heights with respect to $U F$ increased with strain range. The trend of development of both heights was similar with the trend of $R_{\mathrm{a}}$ and $W_{\mathrm{a}}$. A comparison between $R_{\mathrm{a}}$ and the height caused by PSBs showed that these values strongly correlated with each other. A comparison between $W_{\text {a }}$ and the height caused by grain deformation also showed that these values strongly correlated with each other. Consequently, the surface texture parameters $R_{\mathrm{a}}$ and $W_{\mathrm{a}}$ represent the changes in the heights of surface reliefs due to PSBs and grain deformation.
\end{abstract}

\section{Introduction}

When important industrial facilities are subjected to excessive cyclic loadings that lead to deformation, ensuring the structural health of the facilities requires precise damage evaluation from the view-point of material strength. In general, fatigue damage is often assessed using the linear cumulative damage law. However, it is necessary to focus on the physical damage process in order to evaluate the effects of excessive cyclic loadings on subsequent fatigue life more precisely.

The surface of metallic materials become rough during cyclic loading. If the changes in the surface topography can be related to the amount of physical damage, it may be possible to estimate the degree of fatigue damage from measuring surface topography. During cyclic loading, two mechanisms cause the surface roughening of metallic materials: (a) formation of persistent slip bands (PSBs) [1-5] and (b) deformation of crystal grains $[6,7]$. In the PSBs, there are fine peaks and valleys, i.e., extrusions and intrusions caused by active slip systems. The waveforms of these fine reliefs caused by PSBs typically have a wavelength of around 1 $\mu \mathrm{m}$. In contrast, the wavelengths of convex and concave structure due to grain deformation are several times larger than grain size $[6,7]$. Thus, the wavelengths of surface relief due to grain deformation are much larger than those due to PSBs. Several studies have reported that frequency analysis (i.e., the wavelength difference) can be used to separate a PSBs-induced surface relief from a grain-deformation-induced one $[8,9]$.

In our previous study [10], we investigated the change in the surface topography under low-cycle fatigue loadings with constant strain range conditions. The surface relief due to PSBs were separated from those due to grain deformation using frequency analysis and the evolution of each relief was evaluated using two surface texture parameters: arithmetic mean roughness $R_{\mathrm{a}}$ and arithmetic mean waviness $W_{\mathrm{a}}$. As a result, $R_{\mathrm{a}}$ and $W_{\mathrm{a}}$ increased with the increase of usage factor $U F$. The amount of increase rate in $R_{\mathrm{a}}$ and $W_{\mathrm{a}}$ with respect to $U F$ changes with the strain range. Additionally, it suggested that the applied strain range and the degree of fatigue damage $U F$ could be estimated by measuring $R_{\mathrm{a}}$ and $W_{\mathrm{a}}$ if the strain range condition is constant. However, it has not been quantitatively investigated whether the changes in $R_{\mathrm{a}}$ and $W_{\mathrm{a}}$ correspond to the evolution of two surface reliefs due to PSBs and grain deformation.

On the basis of the above background, this study conducted precise observation focusing on PSBs and grain deformations in SUS316NG to quantitatively investigate the cause of the changes in $R_{\mathrm{a}}$ and $W_{\mathrm{a}}$ under low-cycle fatigue loading. During the fatigue tests, the specimen's surface topography was regularly measured using a laser microscope. The surface topographies were analysed by frequency analysis to separate the surface relief due to PSBs from that due to crystal grain deformation. The height caused by PSBs and that by crystal grain deformation were measured respectively.

* Corresponding author: fujimura@eng.hokudai.ac.jp 
To clarify the correspondence between the change mechanisms of surface relief and surface texture parameters, the heights of surface reliefs and surface texture parameters were compared and investigated.

\section{Experimental procedures}

\subsection{Material and specimen}

The material was a solution heat-treated SUS316NG (Nuclear Grade) austenitic stainless steel. The chemical components of the material were $\mathrm{C}$ : $0.01, \mathrm{Si}: 0.40, \mathrm{Mn}$ : 1.70, P: 0.013, S: 0.001, Ni: 12.09 , Cr: 16.64, Mo: 2.48, $\mathrm{N}$ : 0.10 (mass\%). Thermal treatment of the supplied material was undertaken at the temperature of 1050 degrees Celsius for 1 hour, followed by water quenching. The average grain size was $55 \mu \mathrm{m}$. The mechanical properties were: 0.2 proof stress and tensile strength were $\sigma_{0.2}=252 \mathrm{MPa}$ and $\sigma_{\mathrm{B}}=580 \mathrm{MPa}$, respectively. An hourglass-shaped specimen with an R-part of $35 \mathrm{~mm}$ curvature radius and the minimum cross-section diameter of $\phi 6$ was used (Fig. 1). The specimen's surface was mirror finished by polishing with emery paper (grits from 120 to 2000) followed by buffing with diamond abrasives (particle size: $1 \mu \mathrm{m}$ ). To observe the deformation of each grain at the mirror-polished surface, a specimen etched in aqua regia was also prepared.

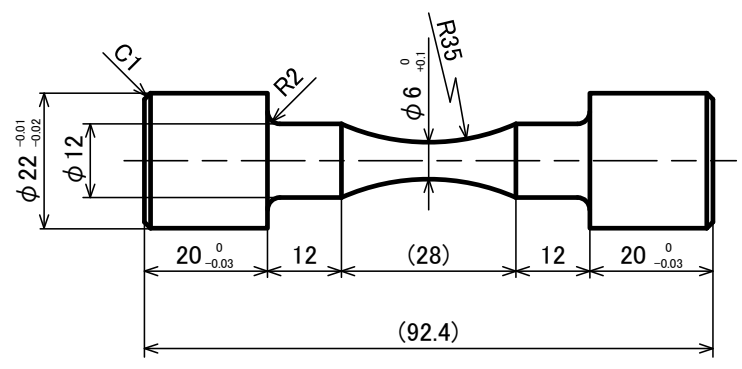

Fig. 1. Schematic of an hourglass-shaped specimen.

\subsection{Fatigue testing method and conditions}

Strain-controlled uniaxial push-pull fatigue tests were conducted using a servo hydraulic fatigue testing machine with a load capacity of $100 \mathrm{kN}$. Triangular loading with constant strain range was applied to specimens in ambient air at room temperature. Axial strain $\varepsilon_{\text {axis }}$ was controlled by measuring the change in diameter at the specimen's minimum diameter part using an extensometer. The strain was calculated using the equation:

$$
\varepsilon_{\text {axis }}=-2 \ln \left(d / d_{0}\right)
$$

where $d_{0}$ is the original diameter at the minimum diameter part, and $d$ is the diameter during the fatigue tests. The constant strain range $\Delta \varepsilon$ were two conditions: $\Delta \varepsilon=4 \%$ and $2 \%$. The strain ratio and the strain rate were $R_{\varepsilon}=-1$ and $0.4 \% / \mathrm{sec}$, respectively.

\subsection{Measurement of surface topography}

During the above-mentioned fatigue tests, the specimen's surface topography was regularly measured using a color 3D laser scanning microscope (VK9700/9710 Generation II, KEYENCE). Cyclic loading was interrupted at arbitrary cycles, and then the specimen was removed from the testing machine for the surface topography measurement. The measurement interval was approximately 0.1 or 0.2 of the usage factor $U F\left(=N / N_{\mathrm{f}}\right)$, which represents the degree of fatigue damage as the consumption rate of life; $N$ is the number of loading cycles, and $N_{\mathrm{f}}$ is the fatigue life. The fatigue life $N_{\mathrm{f}}$ at $\Delta \varepsilon=4 \%$ and $2 \%$ were calculated using the following best-fit curve which were obtained from the fatigue test results in our previous study [11].

$$
\Delta \varepsilon=0.8771 \times N_{\mathrm{f}}^{-0.5043}
$$

3D surface images were taken at four to ten measurement points set on the circumference of the specimen's minimum diameter part. Two object lenses with different magnification were used for the observation of the surface reliefs due to PSBs and grain deformation: one's magnification was 150, and the other's was 20. The number of the high and low magnification images taken at a measurement point was six and one, respectively. The surface topography measurement conditions are summarized in Table 1. Different measurement ranges and resolutions were used for each surface relief because different objective wavelengths were used for the surface topographic images, as mentioned in the next subsection.

Table 1. Surface topography measurement conditions.

\begin{tabular}{|c|c|c|}
\hline Measuring object of surface reliefs & PSBs & Grains \\
\hline Object lens magnification & 150 & 20 \\
\hline Axial measurement range $[\mu \mathrm{m}]$ & 94 & 703 \\
\hline Circumference measurement range $[\mu \mathrm{m}]$ & 70 & 528 \\
\hline $\mathrm{XY}$ resolution $[\mu \mathrm{m} / \mathrm{pixel}]$ & 0.092 & 0.688 \\
\hline $\mathrm{Z}$ resolution $[\mu \mathrm{m} /$ pixel] & 0.02 & 0.02 \\
\hline Wavelength region of filtering $[\mu \mathrm{m}]$ & $0.25-$ & $11.8-$ \\
& 11.8 & 704 \\
\hline
\end{tabular}

\subsection{Image processing method}

To separate the surface topography into the surface relief due to PSBs and that due to grain deformation respectively, the obtained $3 \mathrm{D}$ surface images were analysed by mean of two-dimensional fast Fourier transform (2DFFT) using the image analysis software (VK-Analyzer, KEYENCE).

In the analyses, low-pass and high-pass filters were applied to extract two wavelength range: one from $\lambda_{\mathrm{s}}=$ $0.25 \mu \mathrm{m}$ to $\lambda_{\mathrm{c}}=11.8 \mu \mathrm{m}$ for the surface relief due to PSBs, one from $\lambda_{\mathrm{c}}=11.8 \mu \mathrm{m}$ to $\lambda_{\mathrm{f}}=704 \mu \mathrm{m}$ for that due to grain deformation (Table 1). The filters were brickwall shaped. The $\lambda_{\mathrm{s}}$ and $\lambda_{\mathrm{f}}$ were the cut-off wavelengths to remove measurement noise and the specimen's shape error. These values, $\lambda_{\mathrm{s}}=0.25 \mu \mathrm{m}$ and $\lambda_{\mathrm{c}}=11.8 \mu \mathrm{m}$, 
were determined as they were the ones closest to the JISrecommended value $\left(\lambda_{\mathrm{s}}=0.25 \mu \mathrm{m}\right.$ in JIS B 0651: 2001; $\lambda_{\mathrm{f}}=704 \mu \mathrm{m}$ in JIS B 0610: 2001). The $\lambda_{\mathrm{c}}$ was the cut-off wavelength to separate the surface relief due to PSBs from that due to grain deformation. It was determined to be the shortest wavelength of the surface asperities caused by grain deformation. The shortest wavelength likely corresponds to the length of two adjacent small grains, which rotate opposite to each other. The smallest grain size was estimated to be about $6 \mu \mathrm{m}$ from the grain size distribution of the supplied material, and $11.8 \mu \mathrm{m}$ was chosen for $\lambda_{\mathrm{c}}$ as the value closest to twice the smallest grain size.

\subsection{Height measurement of surface reliefs caused by PSBs and crystal grain deformation}

The height of surface reliefs due to PSBs and that due to grain deformation were respectively measured as follows using the processed surface topographic images.

\subsubsection{Height caused by PSBs}

Fig. 2 shows the processed surface topographic image where PSBs and the surface relief formed on the mirrorpolished specimen's surface were observed. Two measurement lines (light blue lines in (a)) were drawn perpendicular to PSBs (black lines in (a)), and then a profile curve at the intersecting point of the measurement line and PSBs (the red circle in (a)) was obtained as shown in (b). The height due to a PSB was defined as the vertical distance between the maximum peak height and the maximum valley depth in the profile curve of the PSB. Two height data were obtained per one PSB. Five to eight clearly visible PSBs were chosen in one surface topographic image, and the total number of the measured $h$ was from ten to sixteen. Four surface topographic images, taken at different measurement points on the circumference, were used. The measurement interval was approximately 0.1 or 0.2 of $U F$. The $h$ of the same surface relief on PSBs was measured when $U F$ increased in the subsequent loading. The $h$ at $U F=0$ was 0 because PSBs had not appeared yet before cyclic loading.

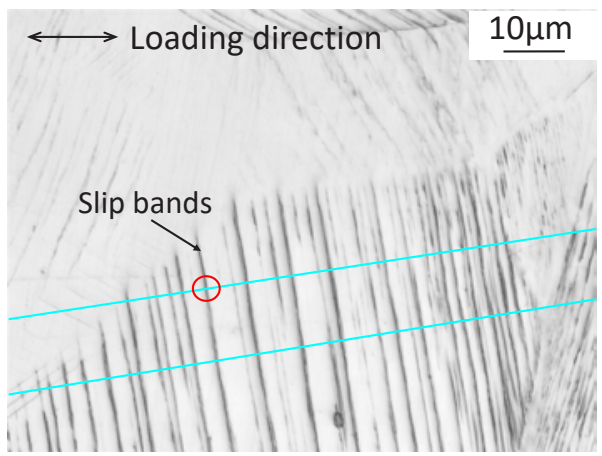

(a) Surface image observed persistent slip bands in grains.

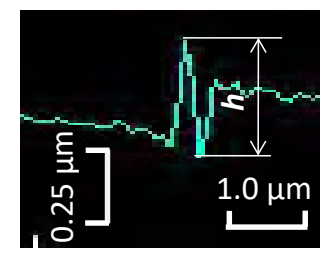

(b) Cross section of a PSB framed by the red circle in (a).

Fig. 2. Measuring method of the height caused by PSBs.

\subsubsection{Height caused by crystal grain deformation}

Fig. 3 shows the processed surface image where crystal grains deformation and the surface relief on the mirrorpolished specimen's surface were observed. Five horizontal measurement lines (light blue lines in (a)) were drawn at an equal interval in the surface image. On the measurement lines, five measurement sections (red lines in (a)) were set at an equal interval: the section length was about twice the average grain size $(110 \mu \mathrm{m})$. A profile curve at the measurement line in the section (the red dotted frame in (a)) was obtained as shown in (b). The height due to grain deformation was defined as the vertical distance between the maximum peak height and the maximum valley depth in the profile curve of the measurement section (the red line in (b)). Twenty five height data were obtained per one surface topographic image. Four surface topographic images, taken at different measurement points on the circumference, were used. The measurement interval was approximately 0.2 of $U F$. The $H$ of the same surface relief on the section was measured when $U F$ increased in the subsequent loading. The $H$ at $U F=0$ was 0 because grains had not been deformed yet before cyclic loading.

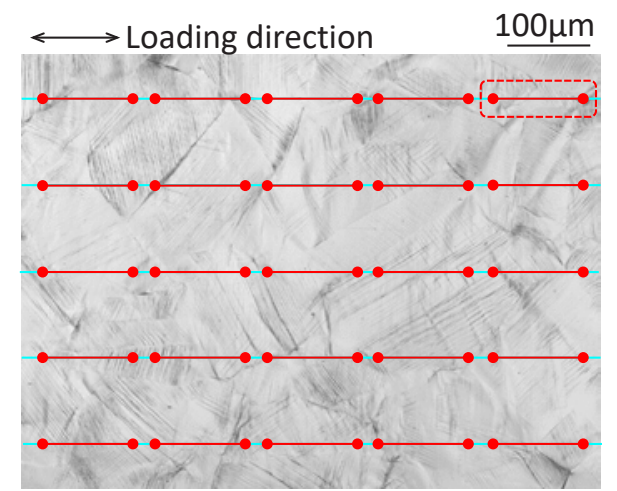

(a) Surface image observed crystal grain deformation.

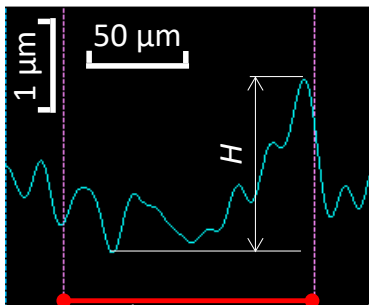

(b) Cross section of the area framed by a red dotted line in (a).

Fig. 3. Measuring method of the height caused by crystal grain deformation. 


\subsection{Calculation of surface texture parameters}

Surface texture parameters $R_{\mathrm{a}}$ and $W_{\mathrm{a}}$ were measured to investigate the correlations between the parameters and the surface reliefs. Areal roughness and waviness parameters (arithmetic mean roughness $R_{\mathrm{a}}$ and waviness $W_{\mathrm{a}}$ ) were determined from the processed surface topographic images, which were used for the height measurement of each surface relief. The area $5 \mu \mathrm{m}$ inward from the edge of the measurement area was excluded for the calculations of $R_{\mathrm{a}}$ and $W_{\mathrm{a}}$. If a crack observed in the measurement area, a region of a few $\mu \mathrm{m}$ surrounding a crack was also excluded. These processes were to prevent abnormal signals affecting the calculation of $R_{\mathrm{a}}$ and $W_{\mathrm{a}}$. Fig. 4 shows the measurement results of surface texture parameters. As mentioned above, $R_{\mathrm{a}}$ and $W_{\mathrm{a}}$ increased with the increase of $U F$, and the amount of increase rate in $R_{\mathrm{a}}$ and $W_{\mathrm{a}}$ with respect to $U F$ changes with the strain range.

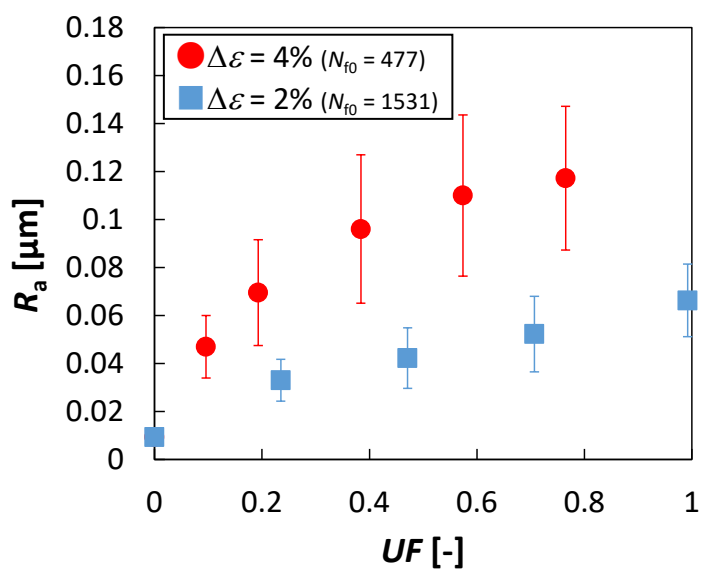

(a) Changes in $R_{\mathrm{a}}$ during cyclic loading.

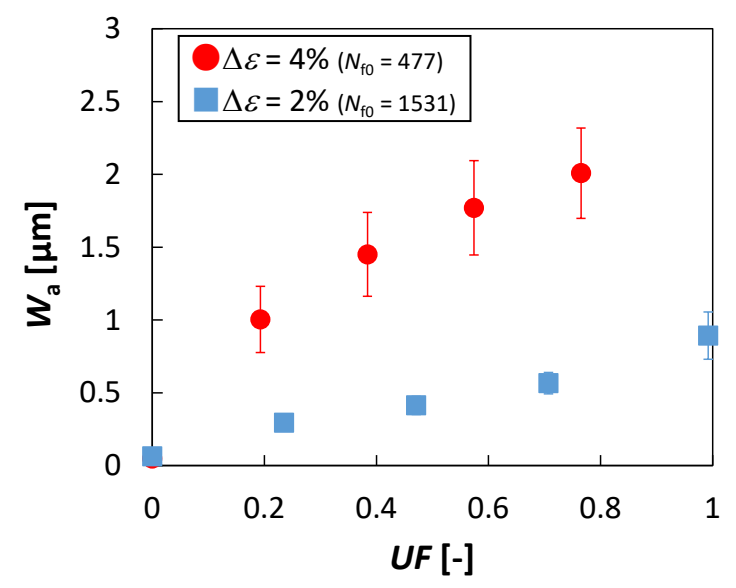

(b) Changes in $W_{\text {a }}$ during cyclic loading.

Fig. 4. Measurement results of surface texture parameters.

\section{Experimental results and discussion}

\subsection{Measurement result of $\boldsymbol{h}$ caused by PSBs}

The measurement results of $h$ are shown in Fig. 5. The vertical and horizontal axes represent $h$ and $U F$, respectively. The circle and square marks indicate the average values of the measurement data at $\Delta \varepsilon=4 \%$ and $2 \%$, and the error bars show the standard deviation. As shown in Fig. 5, the height $h$ rose with the increase of $U F$. Focusing on the increase tendency of $h$, the rate of increase gradually decreases after $U F=0.2$. The amount of increase in $h$ with respect to $U F$ increased with strain range: the $h$ under $\Delta \varepsilon=4 \%$ was larger than that under $\Delta \varepsilon$ $=2 \%$ at the same $U F$.

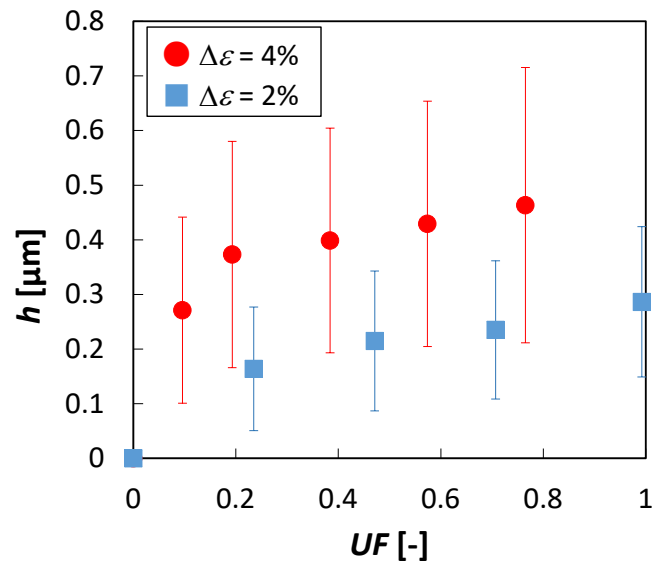

Fig. 5. Measurement results of $h$ caused by PSBs.

\subsection{Relationship between $h$ and $R_{\mathrm{a}}$}

The trend of development of $h$ (Fig. 5) was similar with the trend of $R_{\mathrm{a}}$ (Fig. 4(a)). To clarify the relationship between the height due to PSBs and the surface roughness parameter, $h$ was compared with $R_{\mathrm{a}}$. Fig. 6 shows the scatter diagram: the vertical and the horizontal axes represent $R_{\mathrm{a}}$ and $h$, respectively. Here, $h$ was the average of the measured data per one surface topographic image, and $R_{\mathrm{a}}$ was determined from the image. The circle and square marks indicate the data at $\Delta \varepsilon=4 \%$ and $2 \%$. The black dotted straight line is the regression line, passing through the origin, determined by applying the least-square method to all data on Fig. 6 . As shown in Fig. 6, $R_{\mathrm{a}}$ increased with the increase of $h$. Focusing on the regression line, the coefficient of determination was $\mathrm{R}^{2}=0.8629$, and the correlation coefficient was $\mathrm{R}=0.9289$. The values showed that the height $h$ and surface roughness $R_{\mathrm{a}}$ strongly correlated with each other. Consequently, the surface roughness parameter $R_{\mathrm{a}}$ represents the change in the height of surface relief due to PSBs. 


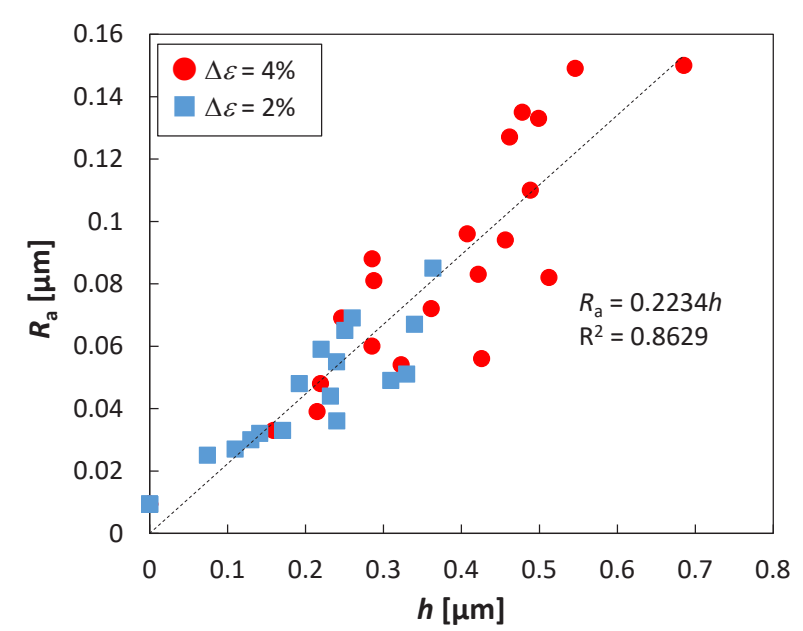

Fig. 6. Relationship between $h$ and $R_{\mathrm{a}}$.

\subsection{Measurement results of $\boldsymbol{H}$ caused by crystal grain deformation}

Figs. 7and 8 show the surface observation of the etched specimen before and after cyclic loading. Grains are visible on the etched surface before cyclic loading (Figs. 7(a) and 8(a)). Figs. 7(b), 7(c), 8(b) and 8(c) show the contour images of the surfaces fatigued under $\Delta \varepsilon=4 \%$ and $2 \%$ : (b) at $U F=0.2$ and (c) at $U F=0.6$. Black lines traced the grain boundaries to define the correspondence between the position of surface relief and grains,. After applying cyclic loading until $U F=0.2$, the convex parts (the red squares in Figs. 7(b) and 8(b)) and the concave parts (the blue dotted square in Figs. 7(b) and 8(b)) were observed on the surface. After more cyclic loading (Figs. $7(\mathrm{c})$ and $8(\mathrm{c}))$, these surface reliefs were developed: the convex part rose higher and the concave part sank deeper; however, their positions on the surface did not change. These convex and concave parts formed at near grain boundaries or within grains, and this observation showed that grain deformation caused the formation of these large surface reliefs.

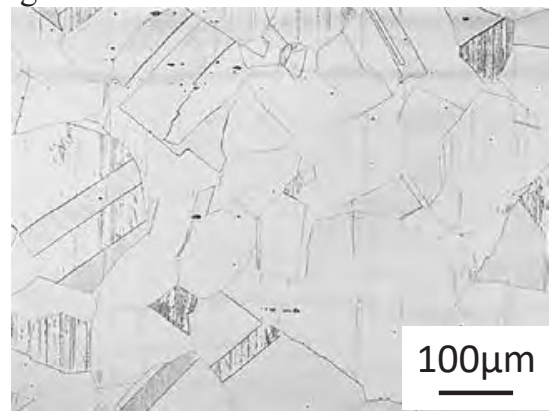

(a) Optical surface image at $U F=0$.

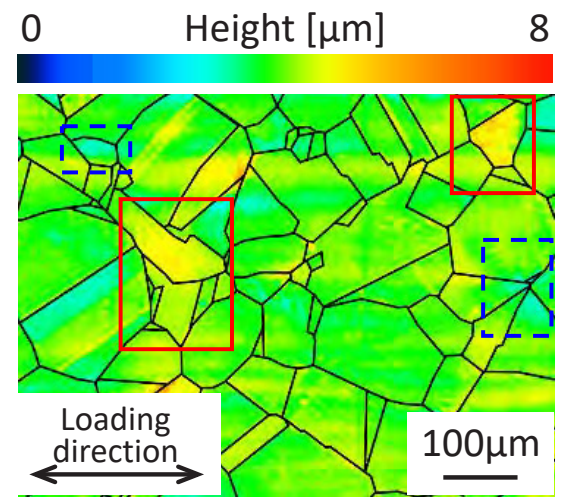

(b) Contour image of the surface fatigued at $U F=0.2$.

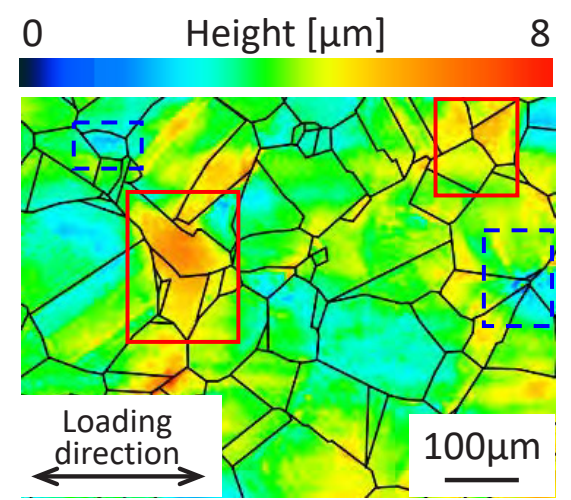

(c) Contour image of the surface fatigued at $U F=0.6$.

Fig. 8. Surface observation of the specimen fatigued under $\Delta \varepsilon=2 \%$. 


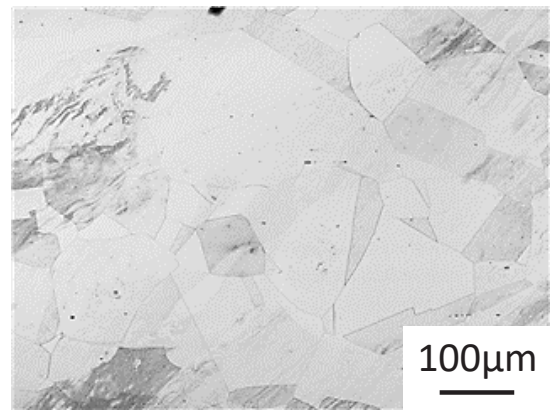

(a) Optical surface image at $U F=0$.

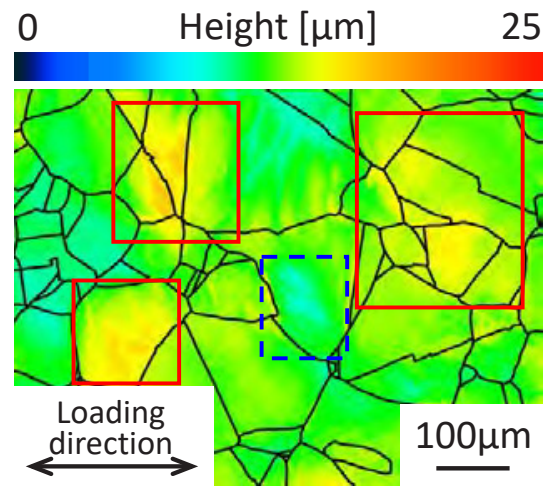

(b) Contour image of the surface fatigued at $U F=0.2$.

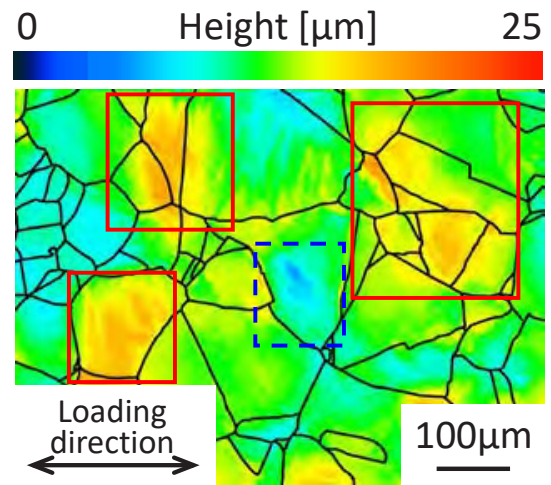

(c) Contour image of the surface fatigued at $U F=0.6$.

Fig. 7. Surface observation of the specimen fatigued under $\Delta \varepsilon=4 \%$.

Fig. 9 shows the measurement results of the height $H$ of the surface reliefs caused by grain deformation. The vertical and horizontal axes represent $H$ and $U F$, respectively. The circle and square marks indicate the average values of the measurement data at $\Delta \varepsilon=4 \%$ and $2 \%$, and the error bars show the standard deviation. As shown in Fig. 9, the height $H$ rose with the increase of $U F$. The amount of increase in $h$ with respect to $U F$ increased with strain range.

\subsection{Relationship between $H$ and $W_{a}$}

The trend of development of $H$ (Fig. 9) was similar with the trend of $W_{\text {a }}$ (Fig. 4(b)). To clarify the relationship between the height due to grain deformation and the surface waviness parameter, $H$ was compared with $W_{\text {a }}$. Fig. 10 shows the scatter diagram: the vertical and horizontal axes represent $W_{\mathrm{a}}$ and $H$, respectively. Here, H

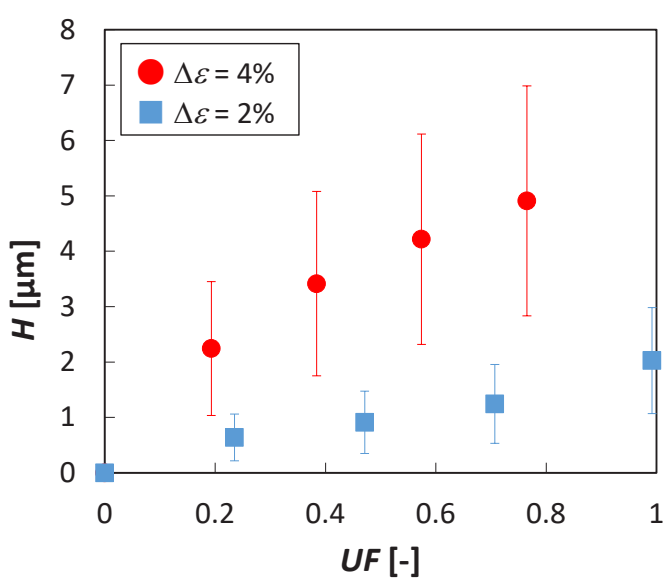

Fig. 9. Measurement results of $H$ caused by grain deformation.

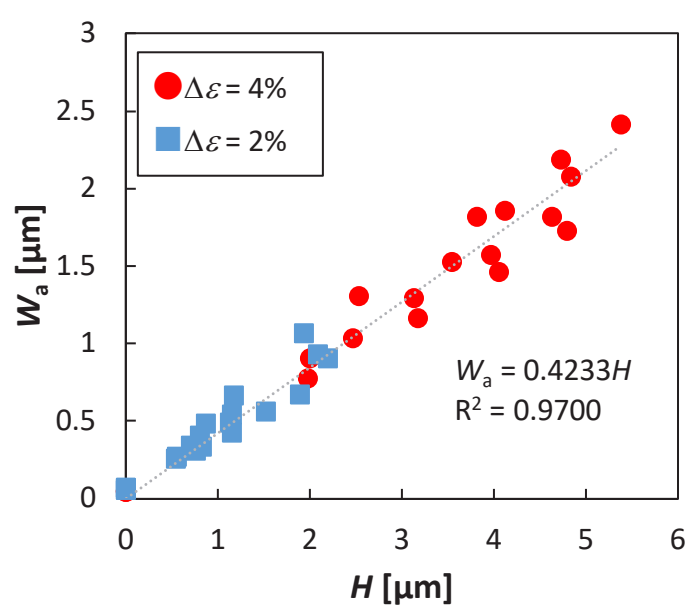

Fig. 10. Relationship between $H$ and $W_{\mathrm{a}}$.

was the average of the measured data per one surface topographic image, and $W_{\mathrm{a}}$ was determined from the image. The circle and square marks indicate the data at $\Delta \varepsilon=4 \%$ and $2 \%$. The black dotted straight line is the regression line, passing through the origin, determined by applying the least-square method to all data on Fig. 10. As shown in Fig. 10, $W_{\text {a }}$ increased with the increase of $H$. Focusing on the regression line, the coefficient of determination was $\mathrm{R}^{2}=0.9700$, and the correlation coefficient was $\mathrm{R}=0.9849$. These values showed that the height $H$ and surface waviness $W_{\text {a }}$ strongly correlated with each other. Consequently, the surface waviness parameter $W_{\mathrm{a}}$ represents the change in the height of surface relief due to grain deformation.

\section{Summary and conclusions}

Precise surface observation focusing on the surface relief due to PSBs and crystal grain deformation were conducted on low-cycle fatigued austenitic stainless steel SUS316NG to quantitatively investigate the cause of the changes in surface texture parameters $R_{\mathrm{a}}$ and $W_{\mathrm{a}}$. During fatigue tests, surface topography was regularly measured using a laser microscope. The height of surface reliefs 
caused by PSBs and grain deformation were measured, respectively. Surface topographies were analysed by frequency analysis to separate the surface relief due to PSBs from that due to grain deformation. The height caused by PSBs and that by grain deformation were measured respectively. The experiments led to the following conclusions:

1. Both heights of surface reliefs rose with the increase of $U F$. The amount of increase in the heights with respect to $U F$ increased with strain range.

2. The trend of development of both heights was similar with the trend of $R_{\mathrm{a}}$ and $W_{\mathrm{a}}$.

3. A comparison between $R_{\mathrm{a}}$ and the height caused by PSBs showed that these values strongly correlated with each other.

4. A comparison between $W_{\mathrm{a}}$ and the height caused by grain deformation also showed that these values strongly correlated with each other.

5. Surface texture parameters $R_{\mathrm{a}}$ and $W_{\mathrm{a}}$ represent the changes in the heights of surface reliefs due to PSBs and grain deformation.

This work was supported by JSPS Grant-in-Aid for Young Scientists (B) Number 17K14552.

\section{References}

1. A. Hunsche, P. Neumann, Acta Matallurgica, 34, 2, 207-217 (1986).

2. P. Villechaise, L. Sabatier, J. C. Girard, Materials Science and Engineering, A323, 377-385 (2002).

3. J. Man, K. Obrtlík, J. Polák, Materials Science and Engineering. A351, 123-132 (2003).

4. J. Polák, J. Man, Materials Science and Engineering. A596, 15-24 (2014).

5. J. Polák, V. Mazánová, M. Heczko, R. Petráš, I. Kuběna, L. Casalena, J. Man, Engineering Fracture Mechanics, 185, 46-60 (2017).

6. I. Shimizu, H. Terai, T. Nosho, T. Abe, Transactions of the Japan Society of Mechanical Engineerings. A64, 620, 1043-1051 (1998).

7. H. Ogawa, K. Hatanaka, Tansactions of the Japan Society of Mechanical Engineerings. A, 68, 665, 5764 (2002).

8. S. Nagaki, T. Abe, E. Ohta, K. Iguchi, Transactions of the Japan Society of Mechanical Engineerings. A, 57, 536, 983-988 (1991).

9. Y. Kakimoto, S. Nagaki, S. Itagaki, T. Abe, Transactions of the Japan Society of Mechanical Engineerings. A, 59, 564, 1888-1894 (1993).

10. T. Shinko, T. Nakamura, N. Fujimura, Y. Nakata, Journal of the Society of Materials Science, Japan, 64, 11, 910-917 (2015).

11. N. Fujimura, T. Nakamura, H. Oguma, Journal of Solid Mechanics and Materials Engineering, 7, 3, 372-380 (2013). 